\title{
Chromatin proteins and modifications as drug targets
}

Helin, Kristian; Dhanak, Dashyant

Published in:

Nature

DOI:

10.1038/nature12751

Publication date:

2013

\section{Document version}

Peer reviewed version

Citation for published version (APA):

Helin, K., \& Dhanak, D. (2013). Chromatin proteins and modifications as drug targets. Nature, 502(7472), $480-8$. https://doi.org/10.1038/nature12751 


\title{
Chromatin proteins and modifications as drug targets
}

\author{
Kristian Helin ${ }^{1,2.3}$ and Dashyant Dhanak ${ }^{4}$
}

${ }^{1}$ Biotech Research and Innovation Centre (BRIC) and ${ }^{2}$ Centre for Epigenetics, University of Copenhagen, Ole Maaløes Vej 5, 2200 Copenhagen, Denmark

${ }^{3}$ The Danish Stem Cell Center (DanStem), University of Copenhagen, Blegdamsvej 3, 2200 Copenhagen, Denmark

${ }^{4}$ Janssen Research \& Development, 1400 McKean Road, P O Box 776, Spring House, PA 19477, USA.

Correspondence: kristian.helin@bric.ku.dk (K.H.) and ddhanak8@ITS.JNJ.com (D.D.) 


\section{Preface (max 100 words)}

A plethora of groundbreaking studies have demonstrated the importance of chromatin-associated proteins and post-translational modifications of histones, proteins and DNA (so-called epigenetic modifications) for transcriptional control and normal development. Disruption of epigenetic control is a frequent event in disease, and the first epigenetic-based therapies for cancer treatment have been approved. A generation of new classes of potent and specific inhibitors for several chromatinassociated proteins have shown promise in pre-clinical trials. Although the biology of epigenetic regulation is complex, these and other new inhibitors will hopefully be of clinical use in the coming years.

\section{Introduction}

Epigenetics is defined as heritable traits not linked to changes in the DNA sequence, however, in more broad terms, epigenetics is used to describe the mechanisms by which chromatin-associated proteins and post-translational modifications (PTMs) of histones regulate transcription. While all cells within an organism contain the same DNA, epigenetic regulators and transcription factors organize the genome into accessible and closed regions, which ensure the correct transcriptional program in a given cell type. Thus, epigenetic regulation is important for maintaining cell identity and is implicated in fundamental processes such as proliferation, development, differentiation and genome integrity. Epigenetic gene regulation can be mediated via DNA methylation, nucleosome remodelling, exchange of histone variants and PTMs of the histones (Box 1). Histones can be modified at specific amino acids with an increasingly diverse set of chemical modifications, such as phosphorylation, acetylation or methylation, ubiquitylation or SUMOylation ${ }^{1,2}$. Research in the last decade has led to a better understanding of the significance of these PTMs. This progress has in particular been achieved through the identification of chromatin-associated proteins that catalyse, recognize and erase the specific modification (Box 1), the generation of high affinity antibodies specific for the post-translational modification, genome-wide location analysis and genetic studies.

Deregulation of epigenetic control is a common feature of a number of diseases including brain disorders and cancer ${ }^{3}$. The involvement of DNA methylation in cancer has been appreciated for a number of years, and the approval of the first drugs targeting DNA methylation is a hallmark for epigenetic-based therapies. The two approved drugs, azacitidine (5-azacytidine) and decitabine (5-aza-2'-deoxycytidine), are nucleoside analogues and irreversible inhibitors of the DNA methyltransferase enzymes, DNMT1 and DNMT3. They are currently used as first-line treatment of 
patients with myelodysplastic syndrome ${ }^{4,5}$. Shortly after the approval of the two DNA methylation inhibitors, the two histone deacetylase (HDAC) inhibitors suberoylanilide hydroxamic acid (SAHA) and Romidepsin (Depsipeptide, FK228) were approved for the treatment of refractory cutaneous Tcell lymphoma ${ }^{6,7}$. Although the introduction of these drugs in the clinic has been a tremendous success for the field, a number of scientific challenges remain. Despite many years of research, we do not understand exactly how and why these drugs work. For the HDAC inhibitors, acetylation is in general increased following drug treatment, however, data demonstrating a correlation between HDAC activity and therapeutic index is still lacking. Similarly, so far there is no established gene expression signature or profile that can predict whether a patient will benefit from the use of HDAC inhibitors. The picture is very similar for the DNMT inhibitors. Although these molecularly targeted drugs have the potential of reverting the epigenetic modification and have been shown to lead to global hypomethylation, we do not know the precise mechanism of action of these drugs. For both classes of drug, the lack of reliable molecular biomarkers for predicting either clinical activity or resistance is a serious drawback limiting the ability to achieve the vision of 'personalized medicine' and despite a large number of clinical trials, the use of the 4 drugs is so far limited to specific haematological cancers.

The recent use of next-generation sequencing technologies on DNA isolated from primary tumours has revealed a high frequency of somatic mutations in genes coding for chromatinassociated proteins known to regulate DNA methylation patterns, histone PTMs and chromatin remodelling (see Ref. 8 for a recent review). Strikingly, the discovery that patients with leukaemia often have mutations in genes such as TET2, IDH1, IDH2 and DNMT3A, which are all involved in regulating DNA methylation patterns, might give insight into why leukaemia patients show a significant response to DNA methylation inhibitors, and in addition could hold promise for future patient stratification strategies. In fact, the lack of genetic data to support the role of chromatinassociated proteins in cancer has been a major obstacle for the development of patient specific targeted therapies. This has dramatically changed with the recent findings that chromatinassociated proteins often show aberrant expression in cancer as a result of translocations and/or genetic amplifications, and now also by the discovery that they carry specific somatic mutations.

In this review, we will focus on the recent advances in the scientific and pharmaceutical communities to develop highly potent and specific inhibitors to chromatin-associated proteins (Table 1). These represent several new classes of therapeutic targets and, as we will exemplify, recent results have shown the feasibility of developing specific inhibitors to histone methyltransferases, histone demethylases, and domains required for the binding of protein 
complexes to specific histone modifications. This is a very exciting time for the field in which the combination of knowledge regarding the role of chromatin-associated proteins in disease and the development of potentially new classes of epigenetic drugs hopefully will lead to molecularly targeted and less toxic therapies with a clear genetic marker(s) for patient stratification.

\section{Targeting Histone Methyltransferases}

An association between histone hypermethylation, transcriptional regulation and the cancer phenotype has spurred efforts to develop specific, small molecule inhibitors of the methyltransferase enzymes involved in histone lysine and arginine methylation. The family of histone methyltransferases (HMTs or more accurately, protein methyltransferases, PR/KMTs) encompasses over sixty different proteins that sequentially transfer a methyl group from the cofactor S-adenosylmethionine (SAM) to the terminal amine of specific substrate lysine and/or arginine residues. With the notable exception of DOT1L (see below), the catalytic transfer of a methyl group from SAM occurs within a conserved SET domain, which accommodates the cofactor and peptide substrates in a conformation conducive for an $\mathrm{S}_{\mathrm{N}} 2$ transfer reaction generating $S$ adenosylhomocysteine (SAH) and the methylated histone side chain as products (Fig. 1). Detailed structural determinations of multiple SET domain containing HMTs have been carried out to support this mechanistic rationale for the methyl transfer event and with a detailed analysis of binding modes of cofactor and/or peptide substrates, to enable the rational design of selective inhibitors. An understanding of exactly how the degree of histone lysine methylation modulates transcription remains to be attained, but the need for the coordinated recruitment of methylation-sensitive proteins to transcriptional complexes offers one plausible hypothesis. Interestingly, the methyltransferases have also been reported ${ }^{9-11}$ to act on various non-histone protein substrates to regulate their functions. However, the relative contributions of histone vs. non-histone action of HMTs are not well understood and continue to be an area of active investigation.

In the cancer context, undoubtedly the discovery of genetic alterations in HMTs in several different tumour types ${ }^{12-14}$ has attracted much attention and provided additional support for the importance of epigenetic deregulation in a disease widely considered to be genetically driven. In some cases (e.g. EZH2 below), heterozygous point mutations in the catalytic SET domain lead to a gain of function of the wild type enzyme $e^{15,16}$ favouring trimethylation and the silencing of tumour suppressor genes and/or differentiation specific genes. Similarly, in other cancers (e.g. NSD2 in multiple myeloma), chromosomal translocations result in increased expression of the methyltransferases again leading to aberrant transcription and proliferation ${ }^{17}$. Conversely, lysine 
methylation induced by the HMT DOT1L results in sustained expression of several genes required for leukemogenesis. Therefore, small molecule inhibitors to for instance EZH2 or DOT1L should have the ability to reduce or eliminate the site-specific lysine methylation introduced by the HMTs and reverse the oncogenic state (see further below).

DOT1L

Chromosomal translocations are relatively common in various hematopoietic malignancies and can be associated with aggressive or poorly responsive disease. In leukaemia involving rearrangement of the Mixed Lineage Leukemia (MLL) gene, translocation leads to fusions with more than 50 different protein partners including ENL, ELL, AF4 and AF9 (Fig. 2a) ${ }^{18}$. The resulting fusion complexes bind to the HMT DOT1L, which specifically methylates the core histone residue histone H3 lysine79 (H3K79) and contributes to transcriptional activation of HOXA10, MEIS1 and other genes required for leukaemia initiation ${ }^{19}$. DOT1L lacks the SET domain commonly present in other lysine methyltransferases but nonetheless can readily catalyse the transfer of one, two or three methyl groups to the $\varepsilon-\mathrm{NH}_{2}$ group of H3K79. In a critical paper from the Armstrong lab ${ }^{20}$, deletion of DOT1L in MLL-rearranged cell lines and subsequently in in vivo mouse studies directly demonstrated the role of the enzyme not only in introducing the H3K79 mark leading to a concomitant increase in gene expression but also in the development of the leukaemia.

Given the significant role of DOT1L in MLL-rearranged leukaemia, inhibitors of its H3K79 methyltransferase activity have been aggressively pursued as potential therapeutics. EPZ004777, a SAM competitive pyrrolopyrimidine derivative (Fig. $2 b, 1)$ was designed ${ }^{21}$ to mimic both SAM and the reaction product SAH whilst also taking advantage of potential hydrophobic interactions available in the binding vicinity. The compound is an extremely potent and remarkably selective SAM-completive inhibitor of the enzyme. In MLL-rearranged cell lines, EPZ004777 reduces global H3K79me2 levels, blocks the expression of MLL-fusion target genes and has antiproliferative activity $^{21}$. Consistent with a targeted mechanism of action, only cell lines with an MLL gene fusion were sensitive to the DOT1L inhibitor whilst non-rearranged lines remained unaffected. Regardless of the measured parameter, the kinetics of cellular response to DOT1L inhibition (and other epigenetic drugs reported so far) is strikingly distinct to the more rapid response usually seen within a few hours with signal transduction modulators (kinase inhibitors) or non-specific chemotherapeutic drugs. Thus, the maximal effect on depletion of the methyl mark is typically seen only after 4-5 days of exposure to drug. Similarly, significant transcriptional changes occur after 6-8 days and $>10$ days are required to observe an antiproliferative phenotype. Defining and 
understanding these distinctive characteristics have important implications for the development of these agents since established measures of biomarker based pharmacodynamic and/or early clinical response may be inappropriate. In addition, prolonged exposure to drug may be required for efficacy further highlighting the need for a selective compound with presumably lower propensity for undesirable off-target effects. Encouragingly, in preclinical experiments, EPZ004777 appeared to be well tolerated when administered to mice at efficacious doses ${ }^{21}$.

Unfortunately, notwithstanding these attractive attributes, poor pharmacokinetics including a short plasma half-life requires EPZ004777 to be administered as a seven day continuous infusion using surgically implanted mini-osmotic pumps. In a preclinical setting, such studies are readily conducted but can pose significant challenges in clinical studies involving cancer patients. In an attempt to address these shortcomings, further modifications of the pyrrolopyrimidine core of the EPZ004777 have been investigated ${ }^{22}$ as an approach to designing second-generation DOT1L targeting drugs. For example, the Structural Genomics Consortium (SGC) has described bromodeaza-SAH (Fig. 2b, 2) as a convenient DOT1L inhibitor allowing for the generation of X-ray co-crystal structures and hence rational design of novel analogues with improved properties ${ }^{23}$. The recent initiation by Epizyme of clinical trials to determine the safety and efficacy of the DOT1L inhibitor EPZ5676 (Ref. 24) in patients with MLL-leukemia is highly significant and represents the first human study of a 'targeted' histone methyltransferase inhibitor.

\section{$E Z H 2$}

Enhancer of Zeste Homolog 2 (EZH2) is the catalytic component of the multi protein polycomb repressive complex 2 (PRC2) and acts as a HKMT at H3K27. Importantly, in cell free systems the EZH2 subunit is only catalytically competent when in a complex with at least two non-enzymatic partners (EED and SUZ12) and moreover in a physiologically relevant, intracellular context, the complex is known to contain two additional proteins (AEBP2 and RBBP4/7) (Fig. 2c) ${ }^{25}$.

PRC2 maintains the transcriptional repression of a large number of genes with key regulatory roles in development and differentiation, and PRC2 proteins are required for normal embryonic development ${ }^{25}$. Pioneering studies from the Chinnaiyan lab have shown an association between increased levels of both EZH2 and H3K27me3 and poor outcomes in metastatic prostate cancer $^{26}$. In addition, inactivating mutations in UTX, a H3K27 demethylase ${ }^{27,28}$ are also similarly correlated suggesting a key role for H3K27 hypermethylation in prostate cancer. Other studies have revealed a similar relationship between elevated levels of EZH2 with silencing of EZH2 target genes and poor prognosis in solid tumours including breast, kidney, and lung ${ }^{29-32}$. More recently, somatic 
activating mutations in the SET domain of EZH2 have been identified in follicular lymphoma (FL), and diffuse large B cell lymphoma (DLBCL), leading to increased H3K27me3 (Refs. 33-35). Taken together, these findings suggest that misregulation of $\mathrm{H} 3 \mathrm{~K} 27$ me3 levels, through EZH2 overexpression or point mutations, silences target genes important in tumour growth and survival and make a compelling case for targeting the enzyme therapeutically. Paradoxically however, inactivating mutations in EZH2 have also been reported in myelodysplastic syndrome $(M D S)^{36}$ raising the potential of a tumour suppressor function for the protein. The role of EZH2 and H3K27 methylation in promoting or inhibiting tumourigenesis and/or maintenance appears therefore to be context dependent and, based on the potential for deleterious effects, suggests caution in developing chronically administered therapeutic inhibitors. Despite these potential drawbacks, multiple pharmaceutical and biotech company research groups have developed highly potent, selective, small molecule inhibitors of EZH2 (Refs. 37-39), and other investigators have pursued equally interesting natural product-based inhibitors ${ }^{40}$.

Medicinal chemistry design of HMT inhibitors has sought to take advantage of the intrinsic affinity of EZH2 for both S-adenosyl-L-homocysteine (SAH, $\left.\mathrm{K}_{\mathrm{i}}=7.5 \mathrm{uM}\right)$ and lysine containing substrate mimetics. Hybrid molecules such as shown in Fig. $2 d(3)$ containing discrete elements of both recognition motifs are modest inhibitors and presumably act as classical bisubstrate inhibitors ${ }^{41}$. However, the relatively low permeability of these highly charged compounds might limit their use in cell-based assays and in vivo. In contrast, despite being devoid of direct EZH2 inhibitory activity, the structurally related and widely used 3-deazaneplanocin (DZNep, Fig. 2d, 4) has been shown to reactivate indirectly PRC2-silenced genes in cancer cell lines by depleting PRC2 subunits ${ }^{42}$. Unfortunately, this activity does not allow for differentiation of selective catalytic inhibition of EZH2 from more global effects of depleting PRC2 including loss of scaffolding functions, microRNA binding sites etc. and, complicates interpretation of cellular phenotypes resulting from true inhibition of H3K27 methylation ${ }^{43}$. Ultimately, the use of DZNep in studies related to investigating the role of EZH2 inhibition in bioassays should be avoided.

High throughput screening of distinct compound libraries by various groups led to the discovery of non-SAM derived catalytic inhibitors of EZH2. Remarkably, all the screens identified compounds with a pyridone amide motif indicating a critical molecular recognition role for the functionality. Although these molecules do not resemble SAM, biochemically they are competitive inhibitors of cofactor binding and various three dimensional homology models have been proposed to rationalize how they may mimic the interactions of the natural substrate. Ultimately, detailed structural studies will be needed to determine unequivocally if both occupy the same binding site in 
EZH2. Despite these uncertainties, extensive chemical modification of the screening hits to improve affinity and pharmaceutical properties led to the discovery of analogues such as shown in Fig. $2 \mathrm{e}^{37} 38$ 39, all highly potent, selective and bioavailable inhibitors of EZH2 in biochemical and cellular assays with in vivo antitumor activity in germinal cell diffuse large B cell lymphomas (DLBCL) with activating EZH2 mutations. Remarkably, these compounds show exquisite selectivity for EZH2 inhibition ( $>10,000$-fold) over most other methyl transferases and can distinguish from EZH1 inhibition ( 100fold). One of these compounds (EPZ-6438, E7438) has entered human clinical trials and several others are likely to follow shortly allowing for an assessment of the therapeutic potential of targeting EZH2 in not only lymphoma but also in solid tumours with $\mathrm{H} 3 \mathrm{~K} 27$ hypertrimethylation. In this context, the recent report of the activity of an EZH2 inhibitor in a preclinical model of paediatric malignant rhabdoid cancer is notable. A subset of these tumours with inactivated SMARCB1 are thought to be dependent on the catalytic activity of EZH2 and in xenograft models, were shown to be sensitive to treatment with the potent and selective EZH2 inhibitor, EPZ6438 (Ref. 44). Interestingly and as mentioned above, other solid tumours (e.g. prostate, breast) have also been associated with dramatic upregulation of EZH2 expression but surprisingly, no convincing data has emerged showing activity of catalytic EZH2 inhibitors in these cancers. As with many other novel potential therapeutics, the safety profile of EZH2 inhibitors remains to be fully defined but initial observations in prolonged animal studies suggest they are well tolerated with little or no overt toxicity and, as mentioned above, E7438 has been advanced to a phase 1/2 clinical trial in patients with advanced solid tumors or with B-cell lymphomas.

\section{Targeting Histone Demethylases}

Previously, methylation was considered to constitute a permanent and irreversible histone modification that defined epigenetic programs in concert with DNA methylation. However, the discovery of, first the lysine-specific demethylase 1 (LSD1, also known as KDM1A, AOF2, BHC110 and KIAA0601) and later the JmjC-domain containing lysine demethylase family has completely changed this view (for recent reviews, see 45,46). LSD1 and its close relative LSD2 (aka KDM1B and AOF1) belong to the superfamily of flavin adenine dinucleotide (FAD)-dependent monooxidases (Fig. 3a). The two proteins can catalyse the demethylation of H3K4me2 and H3K4me1, and LSD1 has in addition been shown to catalyse the demethylation of H3K9me2 and H3K9me1 as well as a number of non-histone proteins such as p53, DNMT1 and E2F1.

The JmjC-domain family 
In contrast to the LSD demethylases, the JmjC-domain containing demethylases can also demethylate tri-methylated lysines. This catalysis involves an oxidative mechanism requiring iron and 2-oxoglutarate as co-factors and most likely occurs through direct hydroxylation of the affected

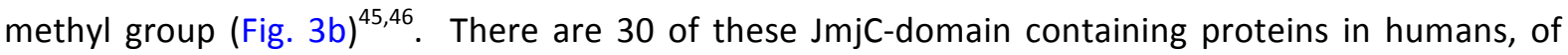
which 17 have been shown to be active histone lysine demethylases. Several results have associated the histone lysine demethylases with disease, in particular cancer and brain disorders. For instance, members of the JMJD2/KDM4 family, which can demethylate H3K9me3/me2 and H3K36me3/me2, have been found overexpressed in squamous cell carcinoma, breast cancer, and medulloblastoma ${ }^{47-}$ 49. Moreover members of the JARID1/KDM5 family that demethylate H3K4me3/me2 are overexpressed in breast and bladder cancers ${ }^{50,51}$, and FBXL10/KDM2B, specific for H3K36me3/me2, is overexpressed in leukaemia ${ }^{52}$. Somatic mutations and deletions have also been identified in the JmjC-domain containing demethylases, including the H3K27me3/me2 demethylase UTX/KDM6A that is found mutated in for instance multiple myeloma and renal cell carcinoma ${ }^{27,28}$, and in JARID1C/KDM5C and PHF8 in X-linked mental retardation patients ${ }^{53,54}$. These mutations often lead to loss of a functional demethylase, and since they may be responsible for the disease phenotype, these observations could suggest that the corresponding histone methyl transferase is a good target for drug development.

Although the understanding of the biological role of the histone demethylases in normal development and disease is still relatively poor, the histone demethylases are considered as attractive targets for drug development due to their association with disease and their well-defined catalytic mechanism. The use of structure-guided design led recently to the first highly potent and selective inhibitors to JmjC-domain containing enzymes ${ }^{55}$. These inhibitors, which are competitive with 2-oxoglutarate and non-competitive with a peptide substrate are potent inhibitors with an $\mathrm{IC}_{50}$ in the nanomolar range, and were shown to be specific for the JMJD3/KDM6B and UTX/KDM6A H3K27 demethylases. JMJD3 has previously been associated with inflammatory responses, and in agreement with this the JMJD3/UTX inhibitor reduced proinflammatory cytokine production by human primary macrophages ${ }^{55}$. In addition to showing the relevance for the catalytic activity of JMJD3 in this process, this study provided proof-of-concept for generating specific JmjC-domain inhibitors. Further proof-of-concept has been provided by the biotech company EpiTherapeutics, which has developed highly potent inhibitors to the JARID1/KDM5 family (Lars-Ole Gerlach, personal communication). These compounds show specific in vivo target engagement of JARID1B, increase in H3K4me3 levels in treated cells and reduced proliferation of cancer cells in a xenograft mouse model [Lars-Ole Gerlach, personal communication]. These proof-of-concept studies provide support for 
that JmjC-domain containing proteins can be targeted by specific compounds, which may have therapeutic applications.

LSD1

It is likely that the first small molecule inhibitors to histone demethylases that go into clinical trials will target LSD1 (Fig. 3c) ${ }^{56}$. Several data have suggested that LSD1 could be an interesting therapeutic target in cancer, because of its high-level expression in prostate cancer, undifferentiated neuroblastoma, oestrogen-negative breast cancer, bladder cancer and colorectal cancer ${ }^{57-60}$. Nevertheless, the recent demonstration that LSD1 is required for the development and maintenance of acute myeloid leukaemia (AML) has gained the most attention ${ }^{61,62}$. Specifically, both genetic and pharmacological data have been provided in vitro and in animal models showing that LSD1 is required to sustain the expression of genes induced by the MLL-AF9 oncoprotein and therefore the maintenance of the leukaemia stem cells $(\operatorname{LSCS})^{61}$. The pharmacological results included the use of the general monooxidase inhibitor tranylcypromine $(T C P)^{62}$, and the TCP-derivative [trans- $\mathrm{N}-((2-$ methoxypyridin-3-yl)methyl)-2-phenylcyclopropan-1-amine)] developed by the biotech company Oryzon (Fig. 3c) ${ }^{63}$, which is more specific and 100 -fold more potent than TCP ${ }^{61}$. The inhibition of LSD1 in AML led to increased differentiation followed by apoptosis, and consistent with this an increase in expression of differentiation markers (e.g. CD11b). The inhibition of LSD1 activity was not associated with a global increase in H3K4me2, however, some increase in H3K4me2 was observed on MLL-AF9 bound genes and genes involved in differentiation ${ }^{61,62}$. Taken together these studies provide proof-of-concept for LSD1 as a therapeutic target in leukaemia, however, the mechanism by which LSD1 contributes to leukaemia is not clear for several reasons. First, LSD1 has been found to be part of several chromatin complexes, including the neuronal silencer co-repressor of RE1-silencing transcription factor (COREST; also known as RCOR1) and the nucleosome remodelling and histone deacetylase (NuRD) ${ }^{45}$ (Fig. 3d). These complexes are found throughout the genome and have a pleotropic role in transcriptional regulation. Second, LSD1 also binds throughout the genome, especially at active promoters and enhancers ${ }^{64,65}$. Third, as mentioned above LSD1 can demethylate both H3K9me2/me1 and H3K4me2/me1 (Fig. 3d). H3K9me2 is normally found associated with repressed chromatin and transcriptional silencing, whereas $\mathrm{H} 3 \mathrm{~K} 4 \mathrm{me} 2 / \mathrm{me} 1$ is associated with active promoters and enhancers. Inhibition of LSD1 activity in AML did not lead to any change in $\mathrm{H} 3 \mathrm{~K} 9 \mathrm{me} 2$, whereas an increase of H3K4me2 was observed on MLL-AF9 target genes ${ }^{61}$ and $C D 11 b^{62}$. These observations raise several questions. First, if LSD1 is bound throughout the genome, why does the inhibition of LSD1 lead to the selective increase of H3K4me2 on specific 
promoters? Second, the expression of MLL-AF9 target genes is decreased in response to LSD1 inhibition, while H3K4me2 is increased. This is counterintuitive, because an increase in H3K4me2 is normally associated with increased expression of a gene, as is the case for CD11b. Therefore, what is the mechanism leading to the decreased expression of MLL-AF9 target genes, and how does inhibition of LSD1 lead to differentiation and apoptosis?

Despite the lack of precise mechanistic insights into the how LSD1 inhibition can lead to inhibition of leukaemia and prolonged survival of mice, the LSD1 inhibitors appear very promising. Oryzon Genomics recently reported on the further development of a clinical compound, ORY-1001, which is more than $1000 x$ more potent than TCP and highly selective over related enzymes, including LSD2 (Ref. 66). The structure of ORY-1001 has not been revealed; however, it has been shown to reduce leukaemic stem cell potential, colony formation, and to induce differentiation of AML cell lines at sub-nanomolar concentrations ${ }^{63}$. Moreover, ORY-1001 leads to the time/dose dependent increase of H3K4me2 at LSD1 target genes (e.g. CD11b) and induction of differentiation markers (Tamara Maes, personal communication). Oryzon Genomics expects to take ORY-1001 into phase I clinical trials later this year.

Interestingly, the potential use of LSD1 inhibitors is not limited to oncological disease. In fact, the weak LSD1 inhibitor TCP has been used as a non-selective monoamine oxidase inhibitor for the treatment of depression ${ }^{67}$, and since aberrant activity of the REST-COREST-LSD complex has been implicated in Huntington's disease ${ }^{68}$ and LSD1 in Herpes infection ${ }^{69}$ the LSD1 inhibitors may also find use for these indications.

\section{Targeting Bromodomains}

Bromodomains constitute a small family of proteins that recognize and bind to acetylated lysine residues on histone tails (Fig. 4a). Acting both as a scaffold for the assembly of larger, multicomponent macromolecular complexes regulating chromatin accessibility as well as for the recruitment of key transcriptional proteins such as RNA polymerase, bromodomain-containing proteins are considered 'readers' of the histone code. The human genome encodes a total of over fifty bromodomain (BRD) proteins, which can be phylogenetically segregated in to eight subfamilies $^{70}$. Embryonic lethality upon genetic knock down of BRDs ${ }^{71}$ underscores the primary importance of the proteins in basic cell function but has also served to limit a better understanding of their role in normal and disease physiology. Structurally, the BRDs are made up of a bundle of four alpha helices joined by two closely interacting but sequence variable loops that form an invaginated, largely hydrophobic pocket for binding to the acetylated lysine ligand ${ }^{70}$. 
The current intense interest in therapeutically targeting various BRDs originated in the demonstration by GlaxoSmithKline (GSK), SGC and Bradner labs that the bromodomain and extraterminal (BET) sub-family ( $\mathrm{Brd2}, \mathrm{Brd} 3, \mathrm{Brd} 4$ and $\mathrm{BrdT}$ ) could be targeted by small molecule antagonists $^{72,73}$. By directly binding to the BET proteins, such compounds prevent the interaction of the reader module to the acetylated histone thereby preventing assembly of an active gene transcriptional complex (Fig. 4a). The ability to disrupt these protein-protein interactions with druglike compounds is remarkable and has been shown in multiple structural studies ${ }^{67}$ to be related to the presence of well defined, deep acetyl lysine binding pockets within the BET proteins. By applying cell based high throughput screening of compound libraries combined with elegant chemoproteomics and a battery of structural and biophysical assays, GSK developed compounds able to inhibit all four BET proteins but with good selectivity over other BRDs. Similarly, SGC working with the Bradner lab developed the widely used JQ1 (Fig. 4b), originating from a patent application by Mitsubishi-Tanabe ${ }^{74}$. Critically, the free availability of these compounds to the research community has dramatically accelerated understanding of the primary mechanism of transcriptional regulation and wider chromatin biology. Indeed, the realization that the pharmacological effects of BET-inhibition could potentially be applied to ameliorate diverse disease phenotypes has spurred further rounds of compound discovery in pharmaceutical companies.

Early evidence for the potential involvement of BET proteins in cancer was the observation that overexpression of Brd2 in lymphocytes induced B-cell lymphomas. Subsequently, French et al reported chromosomal translocation of the Brd4 gene with the NUT protein was the driver for proliferation in the rare but lethal malignancy, NUT-midline carcinoma (NMC) ${ }^{75}$. Further, reversal of the tumour phenotype with BET inhibition provided support not only for the underlying mechanism but also illustrated the therapeutic potential of BET antagonism. Based on this data, a phase I clinical study of the GSK BET inhibitor IBET762 (Fig. 4b), in NMC was initiated in March 2012.

Investigation of the anti-proliferative activity of BET inhibitors in models of hematologic cancer, including AML, Burkitt's lymphoma, multiple myeloma and B-cell acute lymphoblastic leukaemia has revealed perhaps the most exciting facet of BRD biology ${ }^{76,77}$. In these malignancies, BET inhibitors such as JQ1 and the more highly bioavailable IBET151 (Fig. 4b) directly silenced MYC expression via disruption of BET protein binding at the MYC locus. Since the various MYC-isoforms are known to be critical regulators of cell proliferation and survival and MYC is a potent oncogene overexpressed in many cancers, BRD antagonism offers, for the first time, an opportunity generally to target MYC-driven oncogenicity. Intriguingly, however, recent reports have shown critical subtleties in the mechanism of BET inhibitor modulation of $\mathrm{MYC}^{78}$. Whereas in haematological 
cancers, BET regulates C-MYC, in neuroblastoma, BET inhibitor effects appear to be manifested via silencing of N-MYC, presumably by the same or at least a similar mechanism. These results suggest potential for a broader spectrum of activity for BET inhibitors beyond NMC and haematological malignancies and ongoing clinical studies with IBET762 now include other solid tumours such as MYCN-amplified lung and colorectal cancers. The question of a therapeutic window for BET inhibitors in a clinical setting remains to be answered but presumably data from animal toxicity studies did not preclude advancing these compounds to human trials.

Outside of cancer, BET inhibition has shown striking effects in a range of inflammatory disease models suggestive of a central role in lymphocyte lineage aetiology. Interestingly, BET inhibition with IBET762 attenuated only secondary response genes in macrophages with no effect on the primary response elements ${ }^{72}$. The ability to modulate selectively the expression of gene subsets is of significance and raises the possibility of further fine-tuning the level of transcriptional activity with selective inhibitors of other BRDs, which could translate to clinical benefit(s) with fewer undesirable side effects. In mouse models of sepsis, pretreatment with a BET inhibitor suppressed cytokine expression and protected the animals from lethal LPS challenge. In a noteworthy demonstration of activity, administration of the inhibitor even after allergen challenge led to survival $^{69}$. Evidence of the function of other BRDs (SP110, SP140, SMARCA4) in immune-mediated diseases driven by loss of memory $\mathrm{T}$ and B-cells is emerging and limited to tantalizing association of BRD expression and disease phenotype. It is too early to say whether small molecule inhibitors of other BRDs or methyl-lysine readers can be successfully identified, but some promising advances have recently been made with $B A Z 2 B$ and chromodomain proteins associated with brain tumours (Table 1). The development and availability of additional specific small molecule probes will be needed help delineate the biology of these proteins.

\section{Conclusions and perspectives}

This is a very exciting and fruitful time for the "epigenetics field", illustrated with recent discoveries of new classes of enzymes, insights into the biological role of chromatin-associated proteins, findings showing that somatic mutations in genes coding for chromatin-associated proteins are very frequent in cancer, and the development of highly potent and specific small molecule inhibitors to chromatinassociated proteins that show great promise in pre-clinical trials. Until recently, it was uncertain whether it would be technically feasible to generate specific and potent inhibitors to the different classes of readers, writers and erasers of the histone code. However, as discussed in this review, this indeed has been possible for very diverse enzymatic classes, such as the histone methyl transferases, 
the two different subclasses of histone demethylases, and for the non-enzymatic bromodomain containing proteins. These inhibitors are undergoing or will shortly enter human phase I clinical trials for a variety of oncology indications albeit initially in rare tumour types or hematopoietic malignancies.

A major challenge for a potential expansion of the inhibitors to other tumour types will be a better understanding of the mechanism of action of the drugs, and therefore of the biology of the target protein. The ongoing phase I clinical trials have all been designed based on genetic evidence for a role of the targeted protein in the disease (DOT1L in AML, EZH2 in DLBCL, LSD1 in AML and IBET in NUT-midline carcinoma). Such strong genetic evidence does not currently exist in other tumour types, however the effect of the specific inhibitors on large, "omically" well-characterized cell line panels will hopefully help to identify specific genetic alterations that lead to drug sensitivity. Nonetheless, even this approach is unlikely to be straightforward because most chromatinassociated proteins are present in several different multi-component complexes that are associated with several thousand genes and loci throughout the genome. The biology is therefore complex, and depending on the tissue and the underlying genetic landscape of the cell, the chromatinassociated protein could act as an oncogene in one setting but be a tumour suppressor in other circumstances. This is for instance illustrated by EZH2, in which gain-of-function mutations promote lymphoid transformation ${ }^{16,33,37,79}$, and loss-of-function mutations promote MDS and T-ALL ${ }^{36,80-83}$. Similarly, somatic mutations of lysine 27 of H3.3 found in paediatric glioblastoma have been shown to inhibit EZH2 activity ${ }^{84}$. The dual roles of EZH2 and H3K27 methylation might also reflect the biological role of EZH2 and the PRC2 complex. In contrast to signalling pathways and transcription factors, chromatin-associated proteins and epigenetic regulation do not appear to be decisive for lineage choice during differentiation. Instead these proteins are present in the genome to ensure transcriptional patterns and cell identity. In other words, the chromatin-associated proteins often fine-tune transcriptional patterns, and the genes regulated by the proteins can both be oncogenes and tumour suppressor genes. These functions of the chromatin-associated proteins do not mean that inhibitors to these proteins will not have clinical benefit, but highlights the difficulty in identifying biomarkers predictive of tumour sensitivity. This is again illustrated by the EZH2 inhibitors, where the levels of EZH2 in a tumour cell line do not predict whether the cell line will respond to the inhibitor, however, a weak correlation exists between the ability of EZH2 inhibitors (IC50 values) to decrease H3K27me3 levels in DLBCL and inhibition of cell growth ${ }^{37}$.

The generation of small molecule inhibitors to different classes of chromatin-associated proteins has increased not only confidence on the druggability of many epigenetic modulators, but 
has also provided strong insights into the rational design of new compounds with higher affinity and specificity. The hope is that this knowledge can be translated to the generation of specific inhibitors to the many other chromatin-associated proteins involved in cancer. Minimally, such inhibitors will be useful as research compounds to understand the biological function of novel chromatinassociated proteins but could eventually also allow for the identification and therapeutic targeting of other pathways important for the cancer phenotype. Increasingly, it is becoming evident that effective, long term responses to anti-cancer therapies require suppression of two or more oncogenic pathways and this is likely to be the case for epigenetic therapies as well. However, modulation of the cancer epigenome with specific inhibitors may offer unique opportunities to discover effective combination therapies based on the potential directly to alter acquired transcriptional resistance mechanisms. Indeed, a recent report ${ }^{85}$ demonstrating reversal of platinum resistance with HDAC inhibition in ovarian cancer highlights such opportunities. Undoubtedly, other rational combinations remain to be identified and the challenge will be to understand the fundamental cellular alterations induced by epigenetic modulators and to develop complementary agents that synergize most effectively. Along these lines, the resurgence and current success of immunotherapeutic approaches to cancer treatment also offers opportunities for epigenetically targeted therapeutics. In principle, it may be possible to induce cell surface expression of tumour specific antigens allowing for more effective and sustained immune responses to tumours. Finally, the ability to silence critical oncogenes such as MYC and BCL2 with bromodomain inhibitors has been remarkable and unpredicted. Inactivation of these master oncogenic proteins with small molecules has been the Holy Grail for anti-cancer approaches for many years. Yet even here, the lack of a detailed mechanistic understanding of how the BET inhibitors work has led to an empiric approach to determine how best to deploy these agents in the clinic. Despite these limitations, it is important to remember we are nonetheless on the verge of advancing novel molecules with novel biology to human studies with at least some molecular or pathway basis for selecting patients most likely to benefit from these agents. Data from these studies will ultimately determine whether these novel epigenetic therapies will add meaningfully to the armamentarium of the physicians, but the signs

are

promising. 
Table 1 List of small molecule inhibitors to chromatin associated proteins

\begin{tabular}{|c|c|c|}
\hline Chromatin binding protein & Compound & Reference \\
\hline \multicolumn{3}{|l|}{ Histone methyl transferases } \\
\hline \multirow[t]{3}{*}{ DOT1L } & EPZ004777 & 21 \\
\hline & EPZ-5676 & 24 \\
\hline & SGC0946 & 86 \\
\hline \multirow[t]{6}{*}{$\mathrm{EZH} 2$} & GSK126 & 37 \\
\hline & GSK343 & 8788 \\
\hline & EPZ-005687 & 38 \\
\hline & EPZ-6438 & 44 \\
\hline & El1 & 39 \\
\hline & UNC1999 & 89 \\
\hline \multirow[t]{5}{*}{ G9A } & BIX01294 & 90 \\
\hline & UNC0321 & 91 \\
\hline & UNC0638 & 92 \\
\hline & UNC0642 & 88 \\
\hline & BRD4770 & 93 \\
\hline PRMT3 & $14 u$ & 94 \\
\hline \multirow[t]{2}{*}{ PRMT4 (CARM1) } & 17b (BMS) & 95,96 \\
\hline & MethylGene & 97 \\
\hline \multicolumn{3}{|l|}{ Histone demethylases } \\
\hline \multirow[t]{2}{*}{ LSD1 } & Tranylcypromine & 62 \\
\hline & ORY-1001 & 63 \\
\hline JMJD3 & GSK-J1 & 55 \\
\hline \multicolumn{3}{|l|}{ Bromodomains } \\
\hline \multirow[t]{4}{*}{ BET } & JQ1 & 73 \\
\hline & IBET762 & 72 \\
\hline & IBET151 & 76,98 \\
\hline & PFI-1 & 99 \\
\hline BAZ2B & GSK2801 & 88 \\
\hline \multicolumn{3}{|l|}{ Chromodomains } \\
\hline L3MBTL1 & & 100 \\
\hline L3MBTL3 & UNC1215 & 101 \\
\hline
\end{tabular}




\section{Acknowledgements}

Work in the Helin laboratory is supported by the Danish National Research Foundation, the Danish Cancer Society, the Novo Nordisk Foundation, the Lundbeck Foundation, the European Union, The European Research Council, and the Excellence Programme of the University of Copenhagen.

Statement of competing financial interests

K.H. is a cofounder of EpiTherapeutics, is a consultant and has shares and warrants in the company. D.D. is a GSK shareholder and an employee of Jansen Pharmaceuticals. 


\section{References}

1 Berger, S. L. The complex language of chromatin regulation during transcription. Nature 447, 407-412, doi:10.1038/nature05915 (2007).

2 Kouzarides, T. Chromatin modifications and their function. Cell 128, 693-705 (2007).

3 Baylin, S. B. \& Jones, P. A. A decade of exploring the cancer epigenome - biological and translational implications. Nat Rev Cancer 11, 726-734, doi:10.1038/nrc3130 (2011).

4 Issa, J. P. \& Kantarjian, H. M. Targeting DNA methylation. Clin Cancer Res 15, 3938-3946, doi:10.1158/1078-0432.CCR-08-2783 (2009).

5 Yang, X., Lay, F., Han, H. \& Jones, P. A. Targeting DNA methylation for epigenetic therapy. Trends Pharmacol Sci 31, 536-546, doi:10.1016/j.tips.2010.08.001 (2010).

6 Khan, O. \& La Thangue, N. B. HDAC inhibitors in cancer biology: emerging mechanisms and clinical applications. Immunol Cell Bio/ 90, 85-94, doi:10.1038/icb.2011.100 (2012).

7 Wagner, J. M., Hackanson, B., Lubbert, M. \& Jung, M. Histone deacetylase (HDAC) inhibitors in recent clinical trials for cancer therapy. Clin Epigenetics 1, 117-136, doi:10.1007/s13148010-0012-4 (2010).

8 You, J. S. \& Jones, P. A. Cancer genetics and epigenetics: two sides of the same coin? Cancer Cell 22, 9-20, doi:10.1016/j.ccr.2012.06.008 (2012).

9 Huang, J. et al. p53 is regulated by the lysine demethylase LSD1. Nature 449, 105-108, doi:10.1038/nature06092 (2007).

10 Wu, S. C. \& Zhang, Y. Minireview: role of protein methylation and demethylation in nuclear hormone signaling. Mol Endocrinol 23, 1323-1334, doi:10.1210/me.2009-0131 (2009).

11 Zhang, K. \& Dent, S. Y. Histone modifying enzymes and cancer: going beyond histones. J Cell Biochem 96, 1137-1148, doi:10.1002/jcb.20615 (2005).

12 Robinson, G. et al. Novel mutations target distinct subgroups of medulloblastoma. Nature 488, 43-48, doi:10.1038/nature11213 (2012).

13 Ryan, R. J. \& Bernstein, B. E. Molecular biology. Genetic events that shape the cancer epigenome. Science 336, 1513-1514, doi:10.1126/science.1223730 (2012).

14 Shih, A. H., Abdel-Wahab, O., Patel, J. P. \& Levine, R. L. The role of mutations in epigenetic regulators in myeloid malignancies. Nat Rev Cancer 12, 599-612, doi:10.1038/nrc3343 (2012).

15 McCabe, M. T. et al. Mutation of A677 in histone methyltransferase EZH2 in human B-cell lymphoma promotes hypertrimethylation of histone H3 on lysine 27 (H3K27). Proc Natl Acad Sci U S A 109, 2989-2994, doi:10.1073/pnas.1116418109 (2012).

16 Sneeringer, C. J. et al. Coordinated activities of wild-type plus mutant EZH2 drive tumorassociated hypertrimethylation of lysine 27 on histone H3 (H3K27) in human B-cell lymphomas. Proc Natl Acad Sci U S A 107, 20980-20985, doi:10.1073/pnas.1012525107 (2010).

17 Kuo, A. J. et al. NSD2 links dimethylation of histone $\mathrm{H} 3$ at lysine 36 to oncogenic programming. Mol Cell 44, 609-620, doi:10.1016/j.molcel.2011.08.042 (2011).

18 Meyer, C. et al. New insights to the MLL recombinome of acute leukemias. Leukemia 23, 1490-1499, doi:10.1038/leu.2009.33 (2009).

19 Okada, Y. et al. hDOT1L links histone methylation to leukemogenesis. Cell 121, 167-178, doi:10.1016/j.cell.2005.02.020 (2005).

20 Bernt, K. M. et al. MLL-rearranged leukemia is dependent on aberrant H3K79 methylation by DOT1L. Cancer Cell 20, 66-78, doi:10.1016/j.ccr.2011.06.010 (2011).

21 Daigle, S. R. et al. Selective killing of mixed lineage leukemia cells by a potent small-molecule DOT1L inhibitor. Cancer Cell 20, 53-65, doi:10.1016/j.ccr.2011.06.009 (2011). 
Anglin, J. L. et al. Synthesis and structure-activity relationship investigation of adenosinecontaining inhibitors of histone methyltransferase DOT1L. J Med Chem 55, 8066-8074, doi:10.1021/jm300917h (2012).

$23 \mathrm{Yu}, \mathrm{W}$. et al. Bromo-deaza-SAH: a potent and selective DOT1L inhibitor. Bioorg Med Chem 21, 1787-1794, doi:10.1016/j.bmc.2013.01.049 (2013).

24 Daigle, S. R. et al. Potent inhibition of DOT1L as treatment for MLL-fusion leukemia. Blood, doi:10.1182/blood-2013-04-497644 (2013).

25 Morey, L. \& Helin, K. Polycomb group protein-mediated repression of transcription. Trends Biochem Sci 35, 323-332, doi:10.1016/j.tibs.2010.02.009 (2010).

26 Varambally, S. et al. The polycomb group protein EZH2 is involved in progression of prostate cancer. Nature 419, 624-629, doi:10.1038/nature01075 (2002).

27 Dalgliesh, G. L. et al. Systematic sequencing of renal carcinoma reveals inactivation of histone modifying genes. Nature 463, 360-363, doi:10.1038/nature08672 (2010).

28 van Haaften, G. et al. Somatic mutations of the histone H3K27 demethylase gene UTX in human cancer. Nat Genet 41, 521-523, doi:10.1038/ng.349 (2009).

29 Kleer, C. G. et al. EZH2 is a marker of aggressive breast cancer and promotes neoplastic transformation of breast epithelial cells. Proc Natl Acad Sci U S A 100, 11606-11611, doi:10.1073/pnas.1933744100 (2003).

30 Wagener, N. et al. Enhancer of zeste homolog $2(\mathrm{EZH} 2)$ expression is an independent prognostic factor in renal cell carcinoma. BMC Cancer 10, 524, doi:10.1186/1471-2407-10524 (2010).

31 Takawa, M. et al. Validation of the histone methyltransferase EZH2 as a therapeutic target for various types of human cancer and as a prognostic marker. Cancer Sci 102, 1298-1305, doi:10.1111/j.1349-7006.2011.01958.x (2011).

32 Bracken, A. P. et al. EZH2 is downstream of the PRB-E2F pathway, essential for proliferation and amplified in cancer. Embo J 22, 5323-5335, doi:10.1093/emboj/cdg542 (2003).

33 Morin, R. D. et al. Somatic mutations altering EZH2 (Tyr641) in follicular and diffuse large Bcell lymphomas of germinal-center origin. Nat Genet 42, 181-185, doi:10.1038/ng.518 (2010).

34 Pasqualucci, L. et al. Analysis of the coding genome of diffuse large B-cell lymphoma. Nat Genet 43, 830-837, doi:10.1038/ng.892 (2011).

35 Ryan, R. J. et al. EZH2 codon 641 mutations are common in BCL2-rearranged germinal center B cell lymphomas. PLoS One 6, e28585, doi:10.1371/journal.pone.0028585 (2011).

36 Nikoloski, G. et al. Somatic mutations of the histone methyltransferase gene EZH2 in myelodysplastic syndromes. Nat Genet 42, 665-667, doi:10.1038/ng.620 (2010).

37 McCabe, M. T. et al. EZH2 inhibition as a therapeutic strategy for lymphoma with EZH2activating mutations. Nature 492, 108-112, doi:10.1038/nature11606 (2012).

38 Knutson, S. K. et al. A selective inhibitor of EZH2 blocks H3K27 methylation and kills mutant lymphoma cells. Nat Chem Biol 8, 890-896, doi:10.1038/nchembio.1084 (2012).

39 Qi, W. et al. Selective inhibition of Ezh2 by a small molecule inhibitor blocks tumor cells proliferation. Proc Natl Acad Sci U S A 109, 21360-21365, doi:10.1073/pnas.1210371110 (2012).

40 Valente, S. et al. Identification of PR-SET7 and EZH2 selective inhibitors inducing cell death in human leukemia U937 cells. Biochimie 94, 2308-2313, doi:10.1016/j.biochi.2012.06.003 (2012).

41 Epizyme Inc, E. Inhibitors of EZH2, and methods to use thereof. WO2012/034132 (2012).

42 Tan, J. et al. Pharmacologic disruption of Polycomb-repressive complex 2-mediated gene repression selectively induces apoptosis in cancer cells. Genes Dev 21, 1050-1063, doi:10.1101/gad.1524107 (2007). 
Miranda, T. B. et al. DZNep is a global histone methylation inhibitor that reactivates developmental genes not silenced by DNA methylation. Mol Cancer Ther 8, 1579-1588, doi:10.1158/1535-7163.MCT-09-0013 (2009).

44 Knutson, S. K. et al. Durable tumor regression in genetically altered malignant rhabdoid tumors by inhibition of methyltransferase EZH2. Proc Natl Acad Sci U S A 110, 7922-7927, doi:10.1073/pnas.1303800110 (2013).

Kooistra, S. M. \& Helin, K. Molecular mechanisms and potential functions of histone demethylases. Nat Rev Mol Cell Biol 13, 297-311, doi:10.1038/nrm3327 (2012).

Mosammaparast, N. \& Shi, Y. Reversal of histone methylation: biochemical and molecular mechanisms of histone demethylases. Annu Rev Biochem 79, 155-179, doi:10.1146/annurev.biochem.78.070907.103946 (2010).

47 Yang, Z. Q. et al. Identification of a novel gene, GASC1, within an amplicon at 9p23-24 frequently detected in esophageal cancer cell lines. Cancer Res 60, 4735-4739 (2000).

48 Liu, G. et al. Genomic amplification and oncogenic properties of the GASC1 histone demethylase gene in breast cancer. Oncogene 28, 4491-4500, doi:10.1038/onc.2009.297 (2009).

49 Ehrbrecht, A. et al. Comprehensive genomic analysis of desmoplastic medulloblastomas: identification of novel amplified genes and separate evaluation of the different histological components. J Pathol 208, 554-563, doi:10.1002/path.1925 (2006).

50 Lu, P. J. et al. A novel gene (PLU-1) containing highly conserved putative DNA/chromatin binding motifs is specifically up-regulated in breast cancer. J Biol Chem 274, 15633-15645 (1999).

51 Hayami, S. et al. Overexpression of the JmjC histone demethylase KDM5B in human carcinogenesis: involvement in the proliferation of cancer cells through the E2F/RB pathway. Mol Cancer 9, 59, doi:10.1186/1476-4598-9-59 (2010).

52 He, J., Nguyen, A. T. \& Zhang, Y. KDM2b/JHDM1b, an H3K36me2-specific demethylase, is required for initiation and maintenance of acute myeloid leukemia. Blood 117, 3869-3880, doi:10.1182/blood-2010-10-312736 (2011).

53 Jensen, L. R. et al. Mutations in the JARID1C gene, which is involved in transcriptional regulation and chromatin remodeling, cause X-linked mental retardation. Am J Hum Genet 76, 227-236, doi:10.1086/427563 (2005).

54 Laumonnier, F. et al. Mutations in PHF8 are associated with X linked mental retardation and cleft lip/cleft palate. J Med Genet 42, 780-786, doi:10.1136/jmg.2004.029439 (2005).

55 Kruidenier, L. et al. A selective jumonji H3K27 demethylase inhibitor modulates the proinflammatory macrophage response. Nature 488, 404-408, doi:10.1038/nature11262 (2012).

56 Lynch, J. T., Harris, W. J. \& Somervaille, T. C. LSD1 inhibition: a therapeutic strategy in cancer? Expert Opin Ther Targets 16, 1239-1249, doi:10.1517/14728222.2012.722206 (2012).

57 Hayami, S. et al. Overexpression of LSD1 contributes to human carcinogenesis through chromatin regulation in various cancers. Int J Cancer 128, 574-586, doi:10.1002/ijc.25349 (2011).

58 Kauffman, E. C. et al. Role of androgen receptor and associated lysine-demethylase coregulators, LSD1 and JMJD2A, in localized and advanced human bladder cancer. Mol Carcinog 50, 931-944, doi:10.1002/mc.20758 (2011).

59 Kahl, P. et al. Androgen receptor coactivators lysine-specific histone demethylase 1 and four and a half LIM domain protein 2 predict risk of prostate cancer recurrence. Cancer Res 66, 11341-11347, doi:10.1158/0008-5472.CAN-06-1570 (2006). 
Schulte, J. H. et al. Lysine-specific demethylase 1 is strongly expressed in poorly differentiated neuroblastoma: implications for therapy. Cancer Res 69, 2065-2071, doi:10.1158/0008-5472.CAN-08-1735 (2009).

61 Harris, W. J. et al. The histone demethylase KDM1A sustains the oncogenic potential of MLLAF9 leukemia stem cells. Cancer Cell 21, 473-487, doi:10.1016/j.ccr.2012.03.014 (2012). Schenk, T. et al. Inhibition of the LSD1 (KDM1A) demethylase reactivates the all-transretinoic acid differentiation pathway in acute myeloid leukemia. Nat Med 18, 605-611, doi:10.1038/nm.2661 (2012). ORYZON GENOMICS, S. A. Phenylcyclopropylamine derivatives and their medical use. (2010). Ram, O. et al. Combinatorial patterning of chromatin regulators uncovered by genome-wide location analysis in human cells. Cell 147, 1628-1639, doi:10.1016/j.cell.2011.09.057 (2011). Whyte, W. A. et al. Enhancer decommissioning by LSD1 during embryonic stem cell differentiation. Nature 482, 221-225, doi:10.1038/nature10805 (2012). Maes, T. et al. Preclinical characterization of a potent and selective inhibitor of the histone demethylase KDM1A for MLL leukemia. J Clin Oncol, 2013 31, suppl; abstr e13543 (2013). Lee, M. G., Wynder, C., Schmidt, D. M., McCafferty, D. G. \& Shiekhattar, R. Histone H3 lysine 4 demethylation is a target of nonselective antidepressive medications. Chem Biol 13, 563567, doi:10.1016/j.chembiol.2006.05.004 (2006).

68 Zuccato, C. et al. Huntingtin interacts with REST/NRSF to modulate the transcription of NRSE-controlled neuronal genes. Nat Genet 35, 76-83, doi:10.1038/ng1219 (2003). Liang, Y., Vogel, J. L., Narayanan, A., Peng, H. \& Kristie, T. M. Inhibition of the histone demethylase LSD1 blocks alpha-herpesvirus lytic replication and reactivation from latency. Nat Med 15, 1312-1317, doi:10.1038/nm.2051 (2009).

70 Filippakopoulos, P. et al. Histone recognition and large-scale structural analysis of the human bromodomain family. Cell 149, 214-231, doi:10.1016/j.cell.2012.02.013 (2012).

71 Gyuris, A. et al. The chromatin-targeting protein $\mathrm{Brd} 2$ is required for neural tube closure and embryogenesis. Biochim Biophys Acta 1789, 413-421, doi:10.1016/j.bbagrm.2009.03.005 (2009).

72 Nicodeme, E. et al. Suppression of inflammation by a synthetic histone mimic. Nature 468, 1119-1123, doi:10.1038/nature09589 (2010).

73 Filippakopoulos, P. et al. Selective inhibition of BET bromodomains. Nature 468, 1067-1073, doi:10.1038/nature09504 (2010).

74 Mitsubishi-Tanabe Pharma Corporation. Antitumor agent. WO 2009084693 (2009).

75 French, C. A. Demystified molecular pathology of NUT midline carcinomas. J Clin Pathol 63, 492-496, doi:10.1136/jcp.2007.052902 (2010).

76 Dawson, M. A. et al. Inhibition of BET recruitment to chromatin as an effective treatment for MLL-fusion leukaemia. Nature 478, 529-533, doi:10.1038/nature10509 (2011).

77 Delmore, J. E. et al. BET bromodomain inhibition as a therapeutic strategy to target c-Myc. Cell 146, 904-917, doi:10.1016/j.cell.2011.08.017 (2011).

78 Puissant, A. et al. Targeting MYCN in neuroblastoma by BET bromodomain inhibition. Cancer Discov 3, 308-323, doi:10.1158/2159-8290.CD-12-0418 (2013).

79 Beguelin, W. et al. EZH2 Is Required for Germinal Center Formation and Somatic EZH2 Mutations Promote Lymphoid Transformation. Cancer Cell 23, 677-692, doi:10.1016/j.ccr.2013.04.011 (2013).

80 Ernst, T. et al. Inactivating mutations of the histone methyltransferase gene EZH2 in myeloid disorders. Nat Genet 42, 722-726, doi:10.1038/ng.621 (2010).

81 Ntziachristos, P. et al. Genetic inactivation of the polycomb repressive complex 2 in T cell acute lymphoblastic leukemia. Nat Med 18, 298-301, doi:10.1038/nm.2651 (2012). 
Simon, C. et al. A key role for EZH2 and associated genes in mouse and human adult T-cell acute leukemia. Genes Dev 26, 651-656, doi:10.1101/gad.186411.111 (2012).

83 Zhang, J. et al. The genetic basis of early T-cell precursor acute lymphoblastic leukaemia. Nature 481, 157-163, doi:10.1038/nature10725 (2012).

84 Lewis, P. W. et al. Inhibition of PRC2 activity by a gain-of-function H3 mutation found in pediatric glioblastoma. Science 340, 857-861, doi:10.1126/science.1232245 (2013).

Stronach, E. A. et al. HDAC4-regulated STAT1 activation mediates platinum resistance in ovarian cancer. Cancer Res 71, 4412-4422, doi:10.1158/0008-5472.CAN-10-4111 (2011). $\mathrm{Yu}, \mathrm{W}$. et al. Catalytic site remodelling of the DOT1L methyltransferase by selective inhibitors. Nat Commun 3, 1288, doi:10.1038/ncomms2304 (2012).

87 Amatangelo, M. D. et al. Three-dimensional culture sensitizes epithelial ovarian cancer cells to EZH2 methyltransferase inhibition. Cell Cycle 12 (2013).

88 http://www.thesgc.org/scientists/chemical_probes.

89 Konze, K. D. et al. An Orally Bioavailable Chemical Probe of the Lysine Methyltransferases EZH2 and EZH1. ACS Chem Biol, doi:10.1021/cb400133j (2013).

90 Kubicek, S. et al. Reversal of H3K9me2 by a small-molecule inhibitor for the G9a histone methyltransferase. Mol Cell 25, 473-481, doi:10.1016/j.molcel.2007.01.017 (2007).

91 Liu, F. et al. Protein lysine methyltransferase G9a inhibitors: design, synthesis, and structure activity relationships of 2,4-diamino-7-aminoalkoxy-quinazolines. J Med Chem 53, 5844-5857, doi:10.1021/jm100478y (2010).

92 Vedadi, M. et al. A chemical probe selectively inhibits G9a and GLP methyltransferase activity in cells. Nat Chem Biol 7, 566-574, doi:10.1038/nchembio.599 (2011).

93 Yuan, Y. et al. A small-molecule probe of the histone methyltransferase G9a induces cellular senescence in pancreatic adenocarcinoma. ACS Chem Biol 7, 1152-1157, doi:10.1021/cb300139y (2012).

94 Liu, F. et al. Exploiting an allosteric binding site of PRMT3 yields potent and selective inhibitors. J Med Chem 56, 2110-2124, doi:10.1021/jm3018332 (2013).

95 Wan, $\mathrm{H}$. et al. Benzo[d]imidazole inhibitors of Coactivator Associated Arginine Methyltransferase 1 (CARM1)--Hit to Lead studies. Bioorg Med Chem Lett 19, 5063-5066, doi:10.1016/j.bmcl.2009.07.040 (2009).

96 Sack, J. S. et al. Structural basis for CARM1 inhibition by indole and pyrazole inhibitors. Biochem J 436, 331-339, doi:10.1042/BJ20102161 (2011).

97 Allan, M. et al. N-Benzyl-1-heteroaryl-3-(trifluoromethyl)-1H-pyrazole-5-carboxamides as inhibitors of co-activator associated arginine methyltransferase 1 (CARM1). Bioorg Med Chem Lett 19, 1218-1223, doi:10.1016/j.bmcl.2008.12.075 (2009).

98 Seal, J. et al. Identification of a novel series of BET family bromodomain inhibitors: binding mode and profile of I-BET151 (GSK1210151A). Bioorg Med Chem Lett 22, 2968-2972, doi:10.1016/j.bmcl.2012.02.041 (2012).

99 Picaud, S. et al. PFI-1, a Highly Selective Protein Interaction Inhibitor, Targeting BET Bromodomains. Cancer Res 73, 3336-3346, doi:10.1158/0008-5472.CAN-12-3292 (2013).

100 Herold, J. M. et al. Small-molecule ligands of methyl-lysine binding proteins. J Med Chem 54, 2504-2511, doi:10.1021/jm200045v (2011).

101 James, L. I. et al. Discovery of a chemical probe for the L3MBTL3 methyllysine reader domain. Nat Chem Biol 9, 184-191, doi:10.1038/nchembio.1157 (2013).

102 Metzger, E. et al. LSD1 demethylates repressive histone marks to promote androgenreceptor-dependent transcription. Nature 437, 436-439, doi:10.1038/nature04020 (2005).

103 Wissmann, M. et al. Cooperative demethylation by JMJD2C and LSD1 promotes androgen receptor-dependent gene expression. Nat Cell Biol 9, 347-353, doi:10.1038/ncb1546 (2007). 


\section{Highlighted references}

\#3: Excellent overview of epigenetics with an emphasis on the linkage between genomic and epigenomic phenomena in cancer together with opportunities for biomarker driven development of therapeutics.

\#20, \#21. Present compelling evidence for the involvement of DOT1L and H3K79 methylation in MLL-rearranged leukaemia and provide rationale for therapeutic targeting of the enzyme.

\#37. First report of a potent and selective inhibitor of EZH2 with in vitro and in vivo activity vs. EZH2 mutant lymphomas.

\#44. Reports activity of EZH2 inhibitors in solid tumours suggesting potential for clinical benefit beyond haematological malignancies.

\#55. Describes the discovery, structural biology and activity of potent and selective Jumonji demethylase inhibitors.

$\# 61,62$. Highlight the role of LSD1 in AML and the potential for inhibitors to synergize with ATRA therapy.

$\# 72,73,76,77$. Outstanding studies showing demonstrating the feasibility of inhibiting bromodomain proteins in inflammation and tumorigenesis. 


\section{Figure Legends}

Figure 1 Mechanism of lysine methylation catalysed by histone lysine methyltransferases. The lysine amino group of the substrate histone polypeptide engages in an $\mathrm{S}_{N} 2$ reaction with the activated co-factor S-adenosylmethionine (SAM) resulting in the formation of an $\mathrm{N}$-methylated lysine and S-adenosylhomocysteine (SAH).

Figure 2 Histone methyltransferases and inhibitors to DOT1L and EZH2. a, DOT1L catalyses H3K79 methylation of nucleosomes associated with actively transcribed genes. It is recruited by MLL-fusion proteins (here exemplified by MLL-AF10) to MLL-target genes, and is required for leukaemia induced by MLL-fusion proteins. b, Specific inhibitors to DOT1L: EPZ004777 (1, Ref. 21) and Br-SAH (2, Ref. 22). c, PRC2 catalyses di- and trimethylation of $\mathrm{H} 3 \mathrm{~K} 27$ to maintain transcription repression of target genes. These target genes are often associated H3K4me3 as well, a mark of CpG-islands and transcription start sites. d, Reported EZH2 inhibitors: 3 (Ref. 41), DZNep (4, Ref. 42), GSK126 (5, Ref. 37), EPZ7438 (6, Ref. 38) and EI1 (7, Ref. 39).

Figure 3 Histone demethylases and inhibitors to LSD1. a, Reaction mechanism for FAD-dependent LSD1 and LSD2 (modified from Ref. 45). b, Reaction mechanism for JmjC-domain containing histone demethylases (modified from Ref. 45) c, Inhibitors to LSD1. The general monooxidase inhibitor tranylcypromine, and the derivative [trans- $\mathrm{N}-((2-$ methoxypyridin-3-yl)methyl)-2-phenylcyclopropan1-amine)] developed by the biotech company Oryzon (Ref. 63). d, LSD1 is part of several different chromatin complexes, including NURD and COREST, in which it catalyses the demethylation of $\mathrm{H} 3 \mathrm{~K} 4 \mathrm{me} 2 / \mathrm{me} 1$, and as an associated protein with the androgen receptor, together with JMJD2 histone demethylases, where it is responsible for the demethylation of H3K9me2/me1 (Refs. $102,103)$.

Figure 4 Bromodomain proteins and inhibitors to these. a, The Bromodomain can bind to acetylated lysines, which are associated with actively transcribed promoters. The bromodomain proteins (here illustrated by BRD4) have a variety of functions, including mediating the initiation and elongation of transcription. It interacts with $\mathrm{p}$-TEFb, which phosphorylates the C-terminal domain of of RNA polymerase II (Pol II) and induces transcriptional elongation. BRD4 has also been described to interact with a number of protein complexes involved in transcriptional regulation $\mathbf{b}$, Chemical structures of prototypical BET inhibitors. The compounds bind to all members of the BET sub-family 
(Brd2, Brd3, Brd4 and BrdT) with similar affinity and regulate the transcription of key oncogenes including the $M Y C$ family and $B C L 2$.

\section{BOX 1. Post translation modifications of DNA and histones and their role in chromatin} organisation and gene expression.

DNA is wrapped around histones $(\mathrm{H} 2 \mathrm{~A}, \mathrm{H} 2 \mathrm{~B}, \mathrm{H} 3$ and $\mathrm{H} 4)$ to form nucleosomes. Nucleosomes are further compacted to form condensed chromatin. The compaction of DNA is in part regulated via posttranslational modifications (PTMs) of the histone tails, which are protruding from nucleosomes. Epigenetic regulators can in popular terms be divided into writers, readers or erasers of PTMs. The writers comprise enzymes such as histone acetylases, kinases, DNA and histone methyltransferases and ubiquitin ligases. The writers catalyse the PTMs (the epigenetic imprints) on the DNA or the proteins, and may impose epigenetic heritability such as DNA methylation through copying and maintaining the modification. Other modifications, such as histone acetylation, respond rapidly to environmental stimuli and they are therefore more dynamic. Readers of the post-translational modification include proteins with specific domains, such as Bromo-, Chromo-, Tudor-, MBT-, PWWP-, WD40- and PHD-domains, which bind to the specific modification. The readers, which are often found in large protein complexes, interpret the modification and impose changes in chromatin structure. The erasers, such as histone deacetylases (HDACs) and histone demethylases, serve to erase the PTMs and prepare the histones for other modifications. 